DE

M E D I C I N A

T R O P I C A L

$\mathrm{DE}$

S ÃO PAULO

JOURNAL OF THE SÃO PAULO INSTITUTE OF TROPICAL MEDICINE

'Universidade CEUMA, São Luís,

Maranhão, Brazil

${ }^{2}$ Hospital da Criança Dr. Odorico de Amaral Matos, São Luís, Maranhão, Brazil

Correspondence to: Fabrício Silva Pessoa Universidade CEUMA, Departamento de Biologia Microbiana, Rua Josué Montello, 01, Bairro Renascença, CEP 65075-038, São Luís, MA, Brazil

Tel: +55989 8158-3800

E-mail: fabriciosilvapessoa@ hotmail.com

Received: 26 October 2020

Accepted: 26 January 2021

\section{Haemophagocytic lymphohistiocytosis secondary to intrauterine cytomegalovirus infection}

\author{
Fabrício Silva Pessoa ${ }^{(1,2}$, Valdênia Costa Gonçalves ${ }^{(1)}$, Eliza Maria da \\ Costa Brito Lacerda 1
}

\section{ABSTRACT}

Congenital cytomegalovirus infection causes lethal diseases with neurological, visual, auditory and systemic injuries, including the hemophagocytic syndrome. Hemophagocytic lymphohistiocytosis (HLH) can be caused by primary hereditary immunological defects, as well as several infectious triggering factors, such as viruses, bacteria and fungus, among them the cytomegalovirus (CMV). Here we present the case report of a male newborn male, delivered by cesarean at term (gestation age of 39 weeks), weighing 3,250 g, with suffusion skin lesions spread throughout the body, anemia, generalized edema, hepatosplenomegaly, thrombocytopenia associated with grunts and difficulty breathing, treated with ganciclovir after receiving the diagnosis of congenital CMV infection. After a few days of hospitalization, the patient presented with high fever, persistent hepatosplenomegaly and pancytopenia, in addition to elevated ferritin and triglycerides, receiving the diagnosis of HLH treated with immunosuppressive therapy, corticosteroids and intravenous human immunoglobulin. The present case report highlights the importance for health professionals to carry out the investigation of congenital diseases, especially in developing countries, as well as their complications, such as HLH.

KEYWORDS: Cytomegalovirus. Haemophagocytic lymphohistiocytosis. Immunoglobulin.

\section{INTRODUCTION}

Cytomegalovirus (CMV) is a virus of the herpesviridae family that is responsible for the most common and widespread congenital infections worldwide ${ }^{1}$. Congenital $\mathrm{CMV}$ is a global epidemic, and the prevalence of congenital CMV in Brazil is around $0.5-1.1 \%{ }^{1}$. CMV can cause primary infection, primary and persistent infection, reinfection by a different strain or recurrence of the already established infection ${ }^{1}$.

Although the majority of the newborns with CMV infection show no signs of the disease at birth or later, approximately 10-15\% of congenital CMV patients will present symptoms at birth².

Cases of congenital CMV infection present with several signs and symptoms, including petechiae, splenomegaly, hepatomegaly, thrombocytopenia, hepatitis, intrauterine growth restriction that after birth will be perceived as a small for gestational age newborn, central nervous system involvement such as microcephaly, intracranial calcifications, ocular alterations such as chorioretinitis, and sensorineural hearing $\operatorname{loss}^{2}$. However, the literature does not describe HLH associated with intrauterine CMV infection in the neonatal period and the therapeutic protocols for such cases. 


\section{CASE REPORT}

A male newborn was delivered by cesarean section at full-term (39 weeks), weighting 3,250 g and an Apgar score of $8 / 10$ ( $1^{\text {st }}$ and $5^{\text {th }}$ minute of life, respectively) in the city of Sao Joao Batista, Maranhao State, Northeast Brazil. The newborn was admitted to a children's hospital presenting with petechiae and skin suffusions lesions spread throughout the body since birth and associated with grunts and respiratory distress. The mother did not attend pre-natal care. The initial physical examination performed $12 \mathrm{~h}$ after birth revealed the presence of pallor, widespread petechial skin lesions, a scaly palmoplantar rash (Figure 1) and the presence of hepatosplenomegaly in the first hours of life. The newborn was severely ill, had generalized edema but no jaundice. The maternal and neonatal blood groups were not incompatible. Additionally, there was marked microcephaly with a cephalic perimeter of $30.5 \mathrm{~cm}$ for a gestational age of 39 weeks and a birth weight of $3,250 \mathrm{~g}$.

On the $1^{\text {st }}$ day of life, he was admitted to the intensive care unit, with respiratory distress and arterial blood gases showing a moderate metabolic acidosis. The newborn was intubated and placed on mechanical ventilation. A complete blood count was performed which indicated anemia with $6.5 \mathrm{~g} / \mathrm{dL}$ of hemoglobin, leukocytosis (WBC count of $38,900 / \mathrm{mm}^{3}$ ), presence of atypical lymphocytes and thrombocytopenia $\left(45,000 / \mathrm{mm}^{3}\right)$. The chest X-ray was normal.

On the $3^{\text {rd }}$ day of life, serological results showed the presence of IgM and IgG antibodies anti-CMV and treatment with ganciclovir ( $12 \mathrm{mg} / \mathrm{kg} /$ day) was started. A transthoracic echocardiogram was performed, revealing a normal result. Serology was negative for HIV and other TORCH infections, i.e., toxoplasmosis, rubella, zika, herpes simplex, and other organisms including syphilis, parvovirus and varicella zoster.

On the $5^{\text {th }}$ day of life, a computed tomography of the skull showed multiple parenchymal calcifications located in the periventricular region and lentiform nucleus (Figure 1). The retinal scan was normal. There were no cytological or biochemical alterations in the cerebrospinal fluid exam, and the Polymerase Chain Reaction (PCR) for CMV was negative.

On the $7^{\text {th }}$ day of life, the urinary PCR for CMV was positive and the serum quantitative PCR for CMV detected 1.2 million copies per $\mathrm{mL}$ (normal range $<100$ copies $/ \mathrm{mL}$ ).

On the $17^{\text {th }}$ day of life and after 14 days of treatment with ganciclovir and having received one blood transfusion, the clinical and laboratory results worsened and hepatosplenomegaly, pancytopenia and dysthermia were still observed. The newborn underwent a laboratory screening for the haemophagocytic lymphohistiocytosis (HLH) syndrome that revealed the persistence of anemia (hemoglobin $7.4 \mathrm{~g} / \mathrm{dL}$ ), hematocrit $21.9 \%$, and the presence of leukopenia (leukocytes $3,240 / \mathrm{mm}^{3}$ ) with neutropenia $\left(1,340 / \mathrm{mm}^{3}\right)$, atypical lymphocytes and persistence of thrombocytopenia $\left(21,000 / \mathrm{mm}^{3}\right)$. The serum ferritin was extremely high $1,076.80 \mathrm{ng} / \mathrm{mL}$ and elevated triglycerides $370 \mathrm{mg} / \mathrm{dL}$. A bone marrow aspiration was performed, showing a hypocellular and hemodiluted bone marrow, without hemophagocytosis. At that time, the newborn was on assisted ventilatory support, receiving hourly blood transfusions.
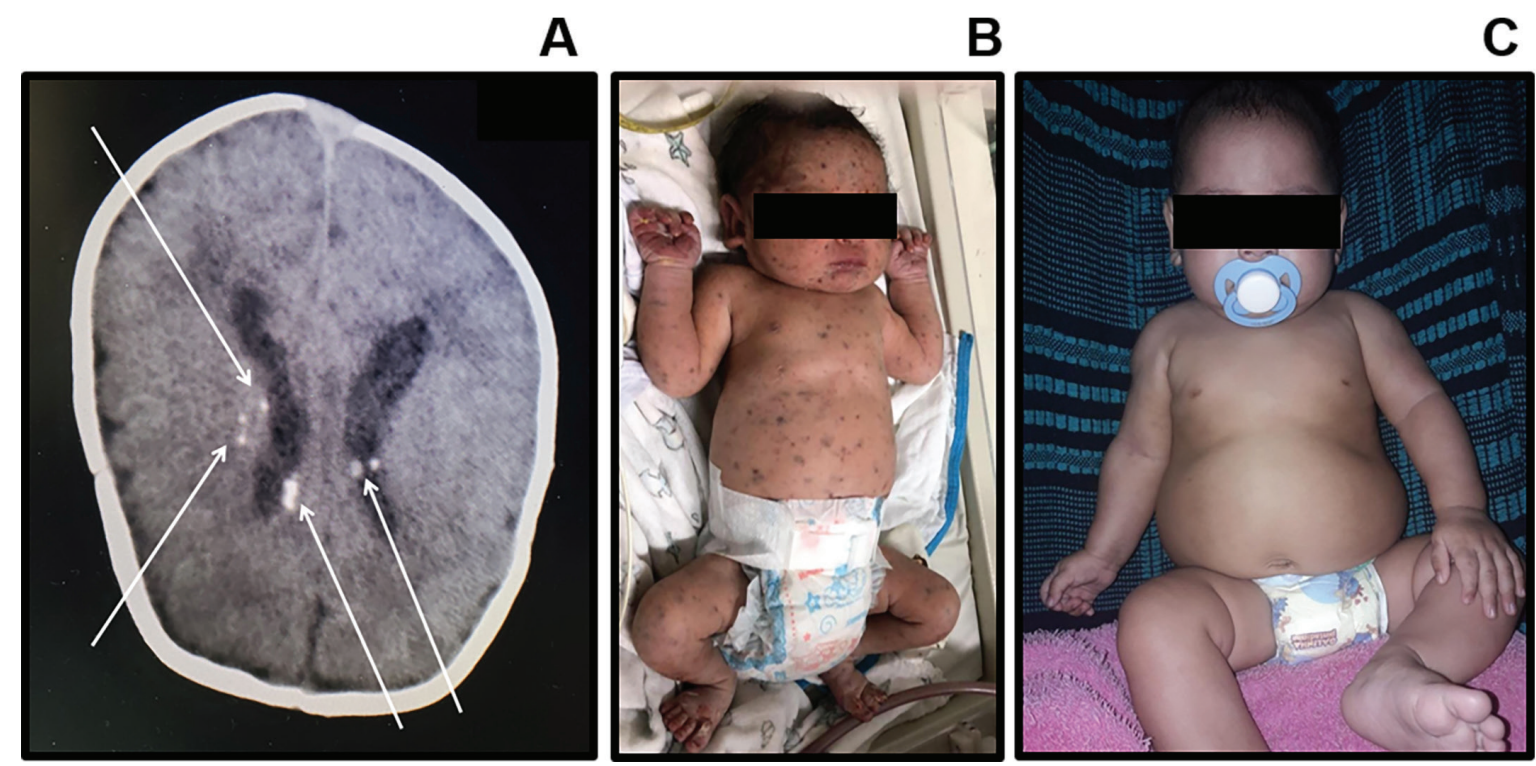

Figure 1 - Initial clinical observation of the newborn (A) Non-contrast computed tomography of the skull shows the presence of calcifications caused by congenital cytomegalovirus infection in a newborn (three weeks old). (B) Characteristic lesions of congenital cytomegalovirus infection in a 12-hours newborn. (C) Infant after seven months of follow-up showing a satisfactory improvement. 
Thus, the newborn fulfilled five criteria for the diagnosis of HLH, two clinical criteria (dysthermia and splenomegaly) and three laboratory criteria (pancytopenia, increased ferritin and increased triglycerides). On the $20^{\text {th }}$ day of life, he was treated with human intravenous immunoglobulin at a dose of $500 \mathrm{mg} / \mathrm{kg} / \mathrm{day}$ for five days and also with corticosteroid therapy (dexamethasone at a dose of $10 \mathrm{mg} / \mathrm{m}^{2}$ daily for two weeks, followed by $5 \mathrm{mg} / \mathrm{m}^{2}$ for two weeks, $2.5 \mathrm{mg} / \mathrm{m}^{2}$ for two weeks and finally $1.25 \mathrm{mg} / \mathrm{m}^{2}$ for two more weeks).

After 10 days of treatment with human intravenous immunoglobulin and corticosteroid, the newborn showed a gradual clinical and laboratory improvement. He was extubated and presented a reduction in serum ferritin level to $338 \mathrm{ng} / \mathrm{mL}$ and triglycerides to $199 \mathrm{mg} / \mathrm{dL}$, a progressive improvement in the leukogram (leukocytosis $8,136 / \mathrm{mm}^{3}$, $7.7 \mathrm{~g} / \mathrm{dL}$ hemoglobin, and platelets $224,500 / \mathrm{mm}^{3}$ ), and a proportional regression of the hepatosplenomegaly with complete remission of the splenomegaly two months after the beginning of the treatment. The subsequent treatment was maintained with dexamethasone for eight weeks. The patient showed clinical and laboratory improvement and was discharged from the hospital for the outpatient follow-up. The child underwent a 6-month follow-up testing at the outpatient clinic for a genetic screening with the collection of cells from the inner region of the infant's mouth to perform DNA extraction. Then, a number of genetic mutations were investigated (PRF1, STX11, STXBP2 and UNC13D), all of which were negative. Because the microcephaly was pre-existent since the intrauterine period, the patient was referred for neuro-psychomotor stimulation and specialized follow-up. Seven months after the diagnosis (Figure 1), the patient still had mild microcephaly and a mild delay in the neuro-psychomotor development, but no visual or auditory losses.

\section{DISCUSSION}

The municipality of Sao Joao Batista has a population of 20,665 inhabitants and is located in the central region of Maranhao State, Northeast Brazil. Since serology for CMV is not part of the prenatal screening and, in addition, it is not a compulsorily notifiable disease, there are no official incidence or prevalence estimates.

Our case presented a complication that has not yet been described in the literature in the neonatal period, i.e., the association of HLH with congenital CMV infection. The patient exhibited a favorable clinical response to the antiviral ganciclovir, aside from the corticosteroid and the intravenous human immunoglobulin therapy.

Unlike the case report described by Silwedel et al. ${ }^{3}$, of a late premature newborn with a birth weight of $1,840 \mathrm{~g}$ and a rare case of secondary HLH triggered by a symptomatic infection (CMV) acquired postnatally, probably transmitted through breast milk, the newborn was treated with ganciclovir without the need of immunosuppressive therapy, while in our case report, the HLH was secondary to intrauterine cytomegalovirus infection, in addition to the successful treatment with intravenous immunoglobulin, corticosteroid and ganciclovir.

The newborn in our case was initially clinically and serologically diagnosed with congenital CMV infection, urinary and serum PCR for CMV, consistent with persistence of an acute CMV infection, treated with ganciclovir. Ganciclovir is the antiviral drug of choice for severe CMV disease ${ }^{4}$.

The urine sample required for the PCR to detect CMVDNA can be collected non-invasively and the viral load in urine can be quantified with this fast, reliable, and specific method $^{5,6}$. In this case, the patient underwent urinary PCR for CMV before starting the treatment with ganciclovir.

After two weeks of antiviral treatment with unsatisfactory clinical response, the newborns's condition worsened with the appearance of dysthermia, no change in hepatosplenomegaly, pancytopenia, and the detection of increased ferritin and triglycerides. These findings fulfilled the criteria of HLH, with the presence of two clinical criteria (dysthermia and splenomegaly) and three laboratory criteria (pancytopenia, hyperferritinemia and hypertriglyceridemia), according to the international criteria for hemophagocytic lymphohistiocytosis-2004. Furthermore, this is in line with the study by Balwierz et $a l .{ }^{7}$ that reported that HLH can occur in all age groups.

Depending on the etiology, HLH can be divided into genetic (primary) and acquired (secondary) forms ${ }^{8}$. The primary cause of HLH has been ruled out, as the genetic tests to detect PRF1, STX11, STXBP2 and UNC13D gene mutations were negative. In this case, HPS was reported as being secondary to a viral infection that has tropism for hematopoietic bone marrow cells ${ }^{9}$. The evaluation of other factors that predispose to HLH, such as the direct antiglobulin test and the investigation of neonatal lupus syndrome were unfortunately not performed in this case.

Hyperferritinemia plays an important role as a marker of disease activity to evaluate therapy response and prognosis. In this case, there was a gradual decrease in the ferritin level after 10 days of treatment ${ }^{9}$. HLH can occur due to genetic causes and secondary causes leading to systemic diseases, while Epstein-Barr virus infection is the main cause in children ${ }^{10}$. Increased ferritin levels and pancytopenia are the most important laboratory abnormalities observed in $\mathrm{HLH}^{10}$. The literature highlights that the anatomopathological 
criterion of HLH may be useful, but is not enough to diagnose the syndrome ${ }^{10}$.

The decision to start therapy for HLH was indicated by the presence of five clinical and laboratory criteria that defined $\mathrm{HLH}$, even without the presence of the bone marrow aspirate criterion. In this case, the newborn was subjected to treatment with intravenous human immunoglobulin and corticosteroids. In the treatment protocol of the second international study of haemophagocytic lymphohistiocytosis proposed in 1994, and revised in 2004 by the Histiocyte Society, for the treatment of primary and secondary HLH forms, they recommended the intravenous human immunoglobulin as a therapeutic alternative. However, in more recent reports such as Chesshyre et al. ${ }^{11}$, the authors described the therapeutic role of intravenous human immunoglobulin and corticotherapy, as therapeutic options of choice for the treatment of secondary HLH, as well as the role of Anakinra, a recombinant and slightly modified version of the human interleukin 1 receptor antagonist protein.

HLH is an excessive and prolonged response to antigen-presenting cells, in this case macrophages and histiocytes, which promote a systemic and potentially lethal inflammatory process. Early diagnosis and immediate treatment is essential to reduce mortality ${ }^{12}$. In this case, the symptoms continued or worsened with the presence of other disease markers. Drug therapy can reduce an exacerbated response, and this patient was treated with human intravenous immunoglobulin (gamma globulin) and dexamethasone, which were fundamental for the infant's clinical and laboratory improvement.

The patient improved satisfactorily with a favorable clinical and laboratory response, reverting the phagocytic activation. Despite the systemic improvement, the microcephaly progressed, however with no hearing loss so far, with a slight delay in the neuro-psychomotor development and monthly follow-ups with a multidisciplinary and a medical team.

\section{CONCLUSION}

Symptomatic congenital CMV infection is a life threatening disease that can progress with neurological and hearing loss and delayed neuro-psychomotor development. However, the description of an HLH case in the neonatal period with a satisfactory clinical response is unprecedented in the literature. This medical condition, although rare, should be considered in all cases of newborns with symptomatic congenital infection.

\section{FUNDING}

No funding.

\section{REFERENCES}

1. Dietrich ML, Schieffelin JS. Congenital cytomegalovirus infection. Ochsner J. 2019;19:123-30.

2. Akpan US, Pillarisetty LS. Congenital cytomegalovirus infection. Treasure Island: StatPearls; 2020. [cited 2021 Jan 26]. Available from: https://www.ncbi.nlm.nih.gov/books/ NBK541003/

3. Silwedel C, Frieauff E, Thomas W, Liese JG, Speer CP. Secondary haemophagocytic lymphohistiocytosis triggered by postnatally acquired cytomegalovirus infection in a late preterm infant. Infection. 2017;45:355-9.

4. Elliot SP. Congenital cytomegalovirus infection: an overview. Infect Disord Drug Targets. 2011;11:432-6.

5. Junqueira JJ, Sancho TM, Santos VA. Citomegalovírus: revisão dos aspectos epidemiológicos, clínicos, diagnósticos e de tratamento. Newslab. 2008;86:88-104.

6. Yamaguchi A, Oh-Ishi T, Arai T, Sakata H, Adachi N, Asanuma $\mathrm{S}$, et al. Screening for seemingly healthy newborns with congenital cytomegalovirus infection by quantitative realtime polymerase chain reaction using newborn urine: an observational study. BMJ Open. 2017;7:e013810.

7. Balwierz W, Czogała M, Pawińska-Wasikowska K, Cwiklińska M, Walicka-Soja K. Hemophagocytic lymphohistiocytosis: diagnostic problems in pediatrics. Przegl Lek. 2010;67:417-24.

8. Malinowska I, Machaczka M, Popko K, Siwicka A, Salamonowicz M, Nasiłowska-Adamska B. Hemophagocytic syndrome in children and adults. Arch Immunol Ther Exp (Warsz). 2014;62:385-94.

9. Hoang MP, Dawson B, Rogers ZR, Scheuermann RH, Rogers BB. Polymerase chain reaction amplification of archival material for Epstein-Barr virus, cytomegalovirus, human herpesvirus 6, and parvovirus B19 in children with bone marrow hemophagocytosis. Hum Pathol. 1998;29:1074-7.

10. Reis PC, Almeida S, Behrens E. Uma nova era no diagnóstico e tratamento da síndrome hemofagocítica. Acta Pediatr Port. 2016;47:333-44.

11. Chesshyre E, Ramanan AV, Roderick MR. Hemophagocytic lymphohistiocytosis and infections: an update. Pediatr Infect Dis J. 2019;38:e54-6.

12. Filipovich AH. Hemophagocytic lymphohistiocytosis (HLH) and related disorders. Hematology Am Soc Hematol Educ Program. 2009;1:127-31.

\section{CONFLICT OF INTERESTS}

The authors declare no conflict of interests. 